\title{
BMJ Open How is rehabilitation with and without an integrated self-management approach perceived by UK community- dwelling stroke survivors? A qualitative process evaluation to explore implementation and contextual variations
}

Fiona Jones, ${ }^{1,2}$ Christopher McKevitt, ${ }^{2,3,4}$ Afsane Riazi, ${ }^{5}$ Matthew Liston ${ }^{1,6}$

To cite: Jones F, McKevitt C, Riazi $A$, et al. How is rehabilitation with and without an integrated selfmanagement approach perceived by UK communitydwelling stroke survivors? A qualitative process evaluation to explore implementation and contextual variations. BMJ Open 2017;7: 014109 . doi:10.1136/bmjopen-2016014109

- Prepublication history for this paper is available online. To view these files please visit the journal online (http://dx.doi.org/10.1136/ bmjopen-2016-014109).

Received 31 August 2016 Revised 26 January 2017 Accepted 6 February 2017

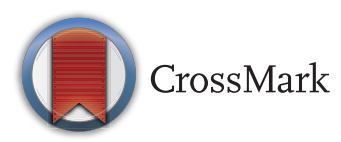

For numbered affiliations see end of article.

Correspondence to Professor Fiona Jones; F.Jones@sgul.kingston.ac.uk

\section{ABSTRACT}

Objective: Self-management programmes could support long-term needs after stroke and using methods integrated into rehabilitation is one option. To explore theoretical assumptions and possible mechanisms of implementation a process evaluation was delivered alongside a cluster trial which has demonstrated feasibility of an integrated self-management programme (Bridges SMP) in community-dwelling stroke survivors. This paper aims to show the extent to which experiences from stroke survivors receiving rehabilitation in control (usual care) and intervention (integrated self-management) sites reflected the differences in rehabilitation received and whether their understandings aligned with the self-management approach employed.

Design: Semistructured qualitative interviews carried out as part of a process evaluation analysed thematically.

Setting: Study was based in South London; all interviews were carried out in participants' home setting.

Participants: 22 stroke participants recruited; 12 from integrated self-management sites and 10 from usual care sites.

Results: All participants revealed shared appreciation of knowledge and support from therapists but subtle differences emerged between sites in respect to perceptions about responsibility, control and how previous experiences were used. Accounts depicted a variance regarding who had structured and planned their rehabilitation, with greater flexibility about content and involvement perceived by participants from the integrated self-management sites. They also provided accounts and experiences which aligned with principles of the intervention, such as self-discovery and problem-solving.

Conclusions: The findings reflect our theoretical assumptions and possible mechanisms of
Strengths and limitations of this study

- This study provided an in-depth understanding of how rehabilitation was perceived by community-dwelling stroke survivors receiving rehabilitation with and without an integrated approach to self-management.

- Interviews were only carried out at one time point and we recognise the views and experiences of participants could change. The findings cannot be used to determine the actual impact or effect of the intervention.

- Use of an independent stakeholder group of stroke survivors helped provide a format for the interviews without making specific reference to the intervention and the term 'self-management' until the end of the interview This is a format that could be used in future trials.

- There is the possibility of personal bias as researchers were not blinded to the allocation of participants.

implementation that rehabilitation with a focus on supporting self-management is reflected in accounts and understandings of stroke survivors. Taken together with our previous research this justifies evaluating the effectiveness of Bridges SMP in a larger sample to further contribute to an understanding of the functioning of the intervention, implementation, contextual factors and mechanisms of impact.

Trial registration number: ISRCTN42534180; Post-results.

\section{BACKGROUND}

Self-management has been defined in various ways but many programmes broadly refer to 
the actions and confidence to manage medical and emotional aspects of a condition to maintain or create new life roles. ${ }^{12}$ In the context of stroke this approach could be advantageous as the adverse emotional impact is high, creating a ripple effect for patients and families alike. ${ }^{3}$ The effects of stroke can also be profound regardless of whether a mild or severe disability results ${ }^{4-6}$ and studies show mood disorders can be experienced by as many as $50 \%$ of all stroke survivors. ${ }^{5}$ Reduced quality of life and social isolation are also common. ${ }^{46}$

If self-management approaches are to be considered a viable way of addressing long-term needs then the nature and organisation of support systems such as stroke rehabilitation also require attention. High levels of support and a focus on professional expertise in the acute phases could paradoxically hamper individuals' confidence and adjustment in the longer term. As some authors have noted if decision-making is made primarily by healthcare professionals, this can restrict individuals from developing personal control and autonomy. ${ }^{7-10}$ While most rehabilitation purports to be patient centred, the level of collaboration in processes such as goal setting can be inconsistent. ${ }^{10}$ In addition, selfmanagement support interventions designed specifically for patients with stroke are relatively rare. Most are currently provided as an 'add-on' to existing services rather than integrated into care. ${ }^{1112}$

Learning from reviews of self-management approaches in other long-term conditions could help devise models relevant to the needs of individuals post stroke. Evidence across the self-management field shows programmes can impact on clinical outcomes and psychological health in patients with a range of long-term conditions. ${ }^{11}{ }^{13}$ Programmes with an emphasis on selfefficacy show most impact compared with weaker effects from didactic methods such as information giving. ${ }^{11}$ However, emerging studies on stroke self-management show programmes are recognised to be theoretically underdeveloped and poorly defined. ${ }^{11}{ }^{12}$ The majority of stroke SMPs have been adapted from generic chronic disease programmes delivered in groups and while they show promising impact on quality of life and functional activity they have yielded mixed results overall. ${ }^{12} 14$ This may be due in part to issues of accessibility and relevance to the different needs of stroke survivors, such as those with communication and cognitive impairments. Despite this a recent study by Cadilhac and colleagues found that those with cognitive, language or global impairments were able to participate in a group-based self-management programme. ${ }^{15}$ Learning how to be inclusive of patients with more complex impairments is an important consideration when designing stroke selfmanagement programmes, since more than one-third of stroke survivors have aphasia. ${ }^{16}{ }^{17}$ Patients with cognitive and communication problems are also more likely to experience depression and may have the most to gain from these approaches. ${ }^{5}$ Therefore programmes which tailor support to individual needs could widen access to more patients with complex conditions.

Introduction of self-management approaches within existing stroke rehabilitation could be one way of addressing unmet needs discussed earlier and with a larger number of stroke survivors. Within healthcare more generally, there is a move towards making every contact count towards behaviour change ${ }^{18}$ and self-management support has the potential to be part of every healthcare interaction. ${ }^{18} 19$ However, this poses an additional challenge by anticipating that healthcare professionals (commonly physiotherapists, occupational therapist and speech and language therapists) can or indeed will integrate self-management strategies within their practice. If supporting self-management and self-efficacy were an explicit and deliberate emphasis of rehabilitation practice, this could potentially impact on issues such as transitions from organised rehabilitation. In addition, self-management approaches integrated into stroke rehabilitation rather than provided as an 'add-on' could offer greater efficiencies within a time limited service.

Bridges stroke self-management programme (SMP) is an individualised approach based on self-efficacy principles designed to integrate into stroke rehabilitation sessions through professional interactions and treatments. ${ }^{20}{ }^{21}$ Professionals are trained to integrate strategies which address defined principles of self-management such as reflection, self-discovery and goal setting. The approach is underpinned by Social Cognitive Theory ${ }^{22}$ and supports self-efficacy through promoting regular feelings of success, described as mastery experiences and values patients' existing expertise. Professionals are encouraged to develop plans and solutions together with patients, as opposed to directing and leading decision-making. This approach is supported by a patient held workbook, which contains vignettes of different stroke survivors describing and reflecting on their self-management solutions. The workbook also has space for users to record and plan their future progress and reflect on their successes and challenges. $^{20}$

Bridges stroke SMP has been developed following the Medical Research Council Framework for the Development and Evaluation of Complex Interventions. ${ }^{23} \mathrm{~A}$ recent cluster trial to evaluate the feasibility of using the SMP within a community stroke rehabilitation team found that it was possible for community-dwelling stroke survivors to be recruited to the trial and receive rehabilitation using defined self-management principles. ${ }^{21}$ The study was not fully powered but demonstrated a change in outcomes which aligned with the underlying theoretical assumptions, that is, an approach based on self-efficacy principles can impact on functional capability post stroke. Bridges SMP is 'by definition' a complex intervention with multiple interacting components, ${ }^{23}$ but we were unsure how rehabilitation with an integrated approach to self-management would be perceived by stroke participants. If there were differences in outcomes how did 
participants in both settings, including usual care sites and sites integrating self-management react to and give meaning to their rehabilitation?

This paper reports findings which are part of a mixed methods approach to evaluating the implementation of an integrated SMP used with community-dwelling stroke survivors. A nested process evaluation was delivered as part of a feasibility cluster trial including (1) nonparticipant observations in which researchers observed therapy but did not interact, (2) qualitative interviews with stroke survivors and (3) focus groups with stroke teams. This paper reports on the qualitative interviews with stroke survivors which aimed to explore (1) whether the experiences and accounts from stroke survivors receiving rehabilitation in control (usual care) and intervention (integrated self-management) sites reflected the differences in the therapy they had received and (2) the extent to which their experiences aligned with some or any of the key principles of the self-management approach used.

\section{METHODS}

\section{Study background and design}

The study reported here is part of a feasibility study to evaluate Bridges Stroke Self-Management Programme (SESAME). Detailed results of the study are described elsewhere. ${ }^{21}$ SESAME comprised a feasibility clusterrandomised design with four community stroke rehabilitation teams as units of randomisation. All stroke participants $(n=78)$ received more than six sessions of community stroke rehabilitation, which either followed usual care (control sites) or usual care integrated with Bridges SMP (intervention sites). Results showed demographic and baseline outcome measures were similar between intervention and control arms, with the exception of age. Overall, Bridges SMP was found to be feasible to deliver within existing services but no outcomes were significantly different between arms. ${ }^{21}$

The nested process evaluation aimed to explore possible mechanisms of implementation in the context of existing services, understand the functioning of the integrated SMP and any contextual variations between sites. ${ }^{24}$ In essence, if there was an impact from the intervention, why might it work? We anticipated the findings could inform future intervention delivery and implementation in a definitive trial. The process evaluation was staged and included three distinct aspects (figure 1)

1. Interviews with stroke survivors from each arm of the trial took place after final 12-week outcome assessments. 2. Non-participant observations of therapy sessions using a fidelity checklist of key principles to explore variation between sites. Observations were carried out between 6-week and 12-week outcome assessments and prior to discharge from rehabilitation. Observations were guided by a fidelity checklist which described seven key principles of Bridges SMP (box 1) (findings to be reported elsewhere).

3. Focus groups were held with intervention and control rehabilitation teams to explore any differences in conceptual understandings of self-management and tangible examples used in rehabilitation practice. Focus groups took place after data collection was completed in full at all sites (to be reported elsewhere).

This paper reports the findings from interviews with stroke participants receiving rehabilitation with and without an integrated approach to self-management, described below.

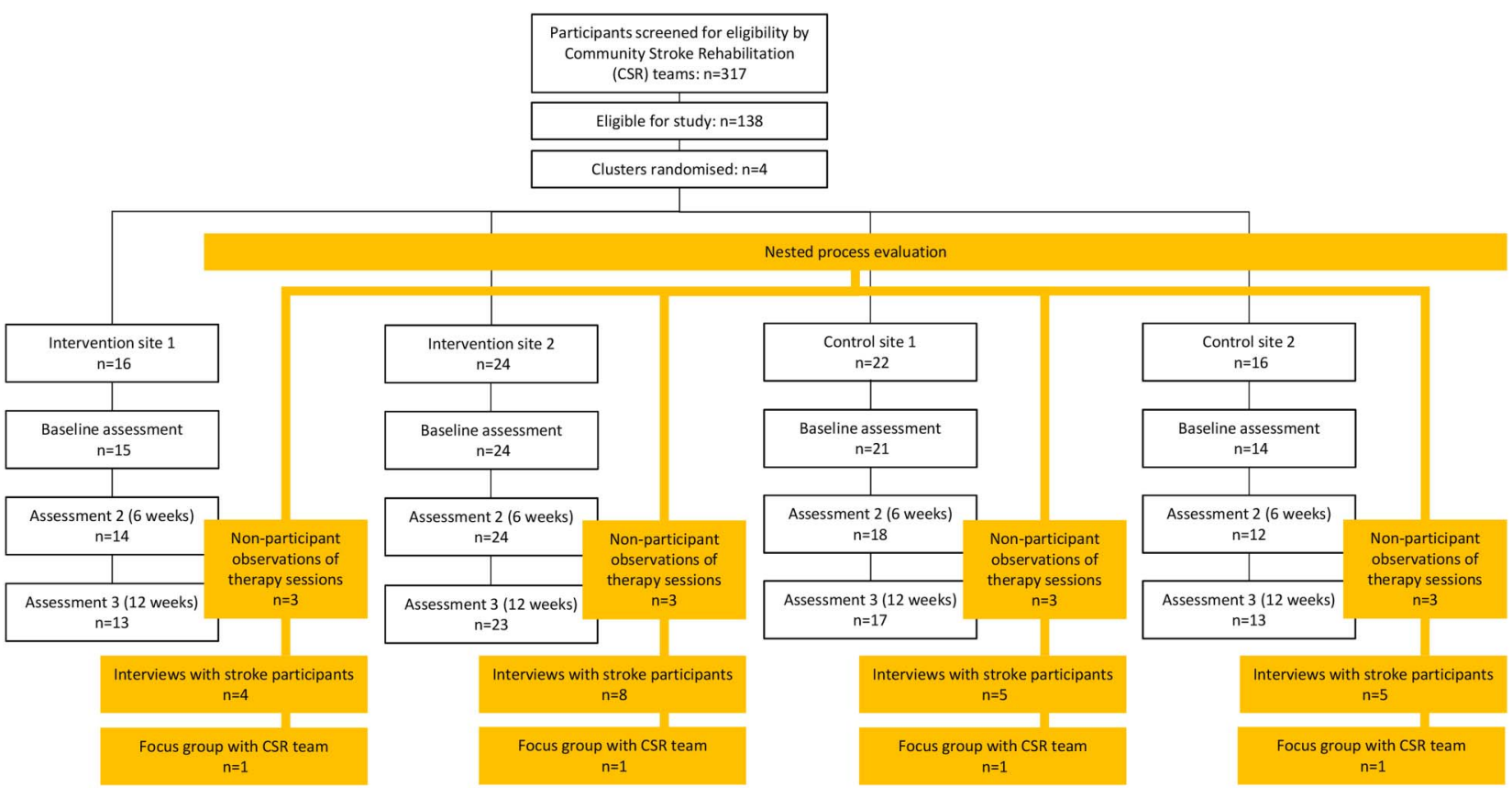

Figure 1 Study flow diagram. 
Box 1 Seven key principles of the Bridges stroke self-management programme

Key Principle

Problem solving

To come up with different ideas, strategies and ways to adjust, rather than relying on suggestions from clinician

Reflection

Attributing changes and progress to personal effort not skills of clinician

Goal setting

Avoiding therapy-led goals, encouraging small steps for mastery experiences and longer term aspirational goals

Accessing resources

To use whatever resources are available to achieve personal goals. Including their own past skills and experiences

Self-discovery

Finding out new ways of doing things and trying out different activities

Activity

Encouraging any activity, however, small

Knowledge

Knowledge about stroke, but also about what works for their own situation and challenges

\section{The integrated self-management intervention}

This study required stroke therapists working in intervention sites to integrate a self-management approach (the intervention) with all recruited stroke participants. The intervention therefore made use of existing scheduled rehabilitation provided by therapists from multidisciplinary community stroke teams. The theoretical assumptions were: if the amount of directed and professionally led therapy was reduced and replaced by therapy focused on supporting self-efficacy and self-management, this would impact on stroke survivors' confidence and functional capability. Figure 2 illustrates a simplified logic model to show structures in place to deliver the intervention, intended activities and outcomes.

As depicted in figure 2, multidisciplinary teams from the intervention (integrated self-management) sites $(n=2)$ undertook 2 days training. This was spread across four, 3-hour sessions to learn ways of adapting their practice and integrating the principles of Bridges SMP including facilitating use of the stroke workbook with participants. Training emphasised underpinning Social Cognitive Theory and self-efficacy, ${ }^{22}$ while at the same time therapists identified and practiced different strategies and techniques to address the intervention principles. Box 1 illustrates the main principles of selfmanagement support used by the intervention teams and illustrative strategies and language for each principle. The aim was to recruit as many stroke survivors with cognitive and communication impairments as possible to reflect usual rehabilitation caseloads. Training included time discussing potential barriers and ways to support self-management incorporating family support if required. Rehabilitation in all sites was delivered by physiotherapists, occupational therapists, speech and language therapists and support workers.

A number of methods were used in training such as role play, case examples and interactive discussions to illustrate to therapists the tangible differences in using a self-management approach compared with their usual practice. Close attention was played to the language used by therapists to engage and support stroke survivors to avoid directive, professionally led practices. The research team and therapists collectively developed a checklist of phrases and strategies that exemplified selfmanagement support for use during rehabilitation sessions.

\section{Study setting and recruitment}

The study took place in London, UK and participants were recruited for one-off interviews from control and intervention cluster sites. Stroke participants were recruited consecutively after their final study assessment from the main sample $(n=78)$ already screened, consented and taken part in the trial. The aim was to gain similar numbers of participants from each of the four research sites and to continue data collection until empirical saturation was reached and no new descriptive codes, categories or themes were generated. All participants had a confirmed diagnosis of stroke, had received more than six treatment sessions from the community stroke rehabilitation team and could follow a two-stage verbal or non-verbal command. Consent to take part in qualitative interviews was obtained by ML.

\section{Data collection}

Qualitative in-depth semistructured interviews were carried out by two senior researchers with clinical rehabilitation experience (FJ, ML) in participants' homes using a topic guide developed with an advisory group consisting of stroke survivors and therapists. The topic guide aimed to explore participants' experience of stroke rehabilitation, the relationship with their therapist and the extent to which they felt confident to selfmanage their progress and everyday life during and after rehabilitation. We were particularly interested about whether the meaning given to rehabilitation by stroke participants aligned with some or any of the key principles of the self-management approach used. Informed by previous research which suggests the word 'self-management' can have negative connotations of being left to get on with it, we avoided using the term in our topic guide. ${ }^{25}$ Together with the advisory group we generated phrases and language to use in interviews which would depict aspects of self-management such as: 'How do you feel about carrying on under your own steam?'; 'Who decided what you would work on in rehabilitation?'; 'Who was in charge of your rehabilitation?' and 'Can you give examples of how you were supported by the therapists?'. These phrases enabled an open discussion about the nature of control and decision-making during rehabilitation, as well as 

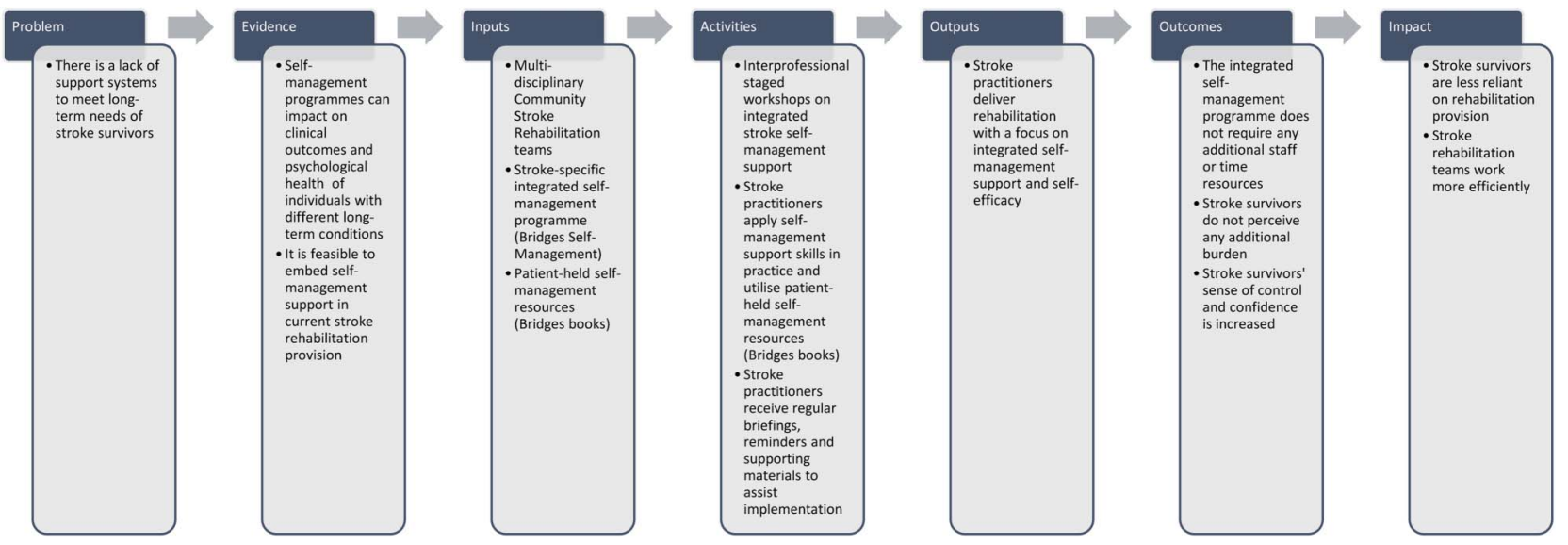

Figure 2 Logic model.

perceived confidence about managing when services finished. We recognised the potential bias of researchers carrying out interviews who were aware of allocation. To mitigate for this we shared initial transcripts with other researchers from a non-clinical background to screen for consistency in questions and contribute their ideas to preliminary analysis.

Interviews lasted between 60 and $90 \mathrm{~min}$ and were audio taped and transcribed verbatim. Field notes were made to capture impressions and any contextual information about the interview, such as whether family members were present.

\section{Data analysis}

An inductive thematic approach was used to capture experiences and accounts from stroke survivors receiving rehabilitation in control and intervention sites and explore issues relating to confidence and control. ${ }^{26}$ Following detailed and close reading all transcripts were systematically and manually coded. A large bank of codes were generated and collated into similar groupings, transcripts were read again a number of times to gather examples of potential themes and thematic maps compiled to clarify their relationship to the central question. Themes were refined several times and discussed between the research team. Refinement continued as each theme was depicted with illustrative quotes taken from across the full data set. Three researchers independently coded transcripts and developed preliminary themes (FJ, CL, SJ). These were discussed and refined with additional input from the research team (CMcK, AR and ML) who coded a sample of transcripts. This process of refinement enabled the progression from descriptive summaries to interpretative themes. A summary of the main findings were sent to all participants.

\section{RESULTS}

For the purpose of reporting the results the control group will be referred to as 'usual care group' and the intervention group as 'integrated self-management group'. The term 'therapist' is used to denote a healthcare professional either a physiotherapist, occupational therapist or speech and language therapist.

A total of 22 participants were recruited consecutively (12 from intervention site and 10 from control sites). There were no refusals to participate or drop outs. There were no differences across groups in ethnicity and social circumstances and ages ranged from 27 to 92 years. All participants were interviewed in their homes and five had family members present. Voices of family were transcribed but not included in the analysis. Study participants had similar levels of mood, functional capacity, quality of life and self-efficacy. Analysis identified patterns including similarities and differences across the transcripts in relation to the meaning given to rehabilitation. Generation of themes was driven by the data and the ways in which participants construed their experiences, relationships with therapists, reflections and the types of examples used. Two overarching themes were prevalent across the entire data set but included a number of subthemes which made distinctive contributions to different facets of the theme. All subthemes contributed to the wider themes of (1) control and responsibility for progress and (2) knowledge and resources. However, some subthemes were developed entirely from either usual care or integrated selfmanagement group participants, for example, the subtheme 'Importance of doing things the right way' was formed entirely by responses from participants in the usual care group. To reflect the differences and similarities of accounts, the derivation of illustrative quotes; that is, whether they are from integrated selfmanagement or usual care sites is given along with participant code.

\section{Theme 1-control and responsibility for progress}

This theme highlights the ways in which experiences varied in terms of how participants perceived who controlled their rehabilitation. There was general confirmation in all transcripts of the importance of 
rehabilitation and its inextricable link to progress. However participants in the integrated self-management sites often inferred that responsibility for progress was shared between both parties, whereas participants in the usual care group often identified the therapist as being pivotal to their progress. Participants gave different accounts about who made the decisions and who decided what was most important to focus on. Four subthemes contributed to the overall concept of whether further progress was controlled by the stroke participant, the therapist or both and comprised (1) Who establishes the plan, (2) Varying importance of physical markers of progress, (3) Importance of doing things the right way and (4) What happens in the future?

\section{Who establishes the plan?}

There seemed to be notable variations in how participants recalled ways in which they were first told what to expect and what rehabilitation could achieve. Some felt rather detached from how 'the plan' for their rehabilitation was established; the sense of being guided along a path by the therapist was evident in many accounts given by usual care participants.

She seemed like she had got a plan when she came and she was gonna do her plan. (usual care 12)

They don't ask, they just tell me what they want me to do and I just do it (usual care 8)

Participants gave examples of the type of judgments given by therapists which formed the foundation for session content and priorities chosen.

They just came in and said let's see how much you can do. Toast- can you spread the butter? or can you go up and down the stairs?. He said go to the garden and walk back and that, to see a mistake or look at how my balance is. (usual care 11)

However, some early formations of 'the plan' were generated from a series of questions posed by the therapist which helped to jointly establish priorities and areas of attention, participants described feeling listened to and having affirmation of their ideas and goals.

The very first session was what do you hope to achieve? She asked me 'what do I want to get out of therapy?' (integrated self-management 4)

In general the first encounters with therapists described by participants were ascribed a singular importance, often unquestioned and could set the scene for subsequent rehabilitation sessions. Aligned with this were reflections concerning what success 'looked like' in rehabilitation sessions and the markers for progress given by therapists, for example, when a participant was told how much quicker they were at walking or how much they were able to balance.
Varying importance of physical markers of progress

This subtheme was developed entirely from accounts from usual care site participants who gave many more examples of how therapists had used their own varied physical markers; for example, relating to speed or number of exercises carried out as a way of distinguishing between success and failure.

She said right I want you to get from that settee, stand up go over there and walk back. And she'd say that's not bad that's 21 seconds. (usual care 12)

She went through these sorts of things to find out if I could- and look at the improvements as I went along. (usual care 9)

Often markers of progress such as distance walked were recorded by the therapists in their professional notes and contributed towards a construction of 'success' based on physical markers and the opinions and ratings given by their therapist.

She would say that she was impressed that the target was achieved. So she was happy about giving me targets. (usual care 10)

One participant talked about not realising how 'bad' they were until they were shown by the therapist.

There's a lot of things she told me and its upsetting methinking that I'm really good and then she says right we are going to do this and I can't do it. (usual care 12)

The way in which participants recalled the physical markers used by therapists was not necessarily perceived negatively as implied in the quote above, they were also given as examples of affirmation about progress and effort.

\section{Importance of doing things the right way}

Linked closely with the previous subtheme, there was meaning given by accounts from all usual care site participants of doing things the 'right way'. Some gave examples of how they were told by therapists to avoid too much experimentation in case it was detrimental to recovery.

She said oh you shouldn't be doing that. There's actually some things you shouldn't be doing and you'll actually make it worse. (usual care 12)

I didn't want to do the wrong thing by overdoing it or under doing it. (I needed) confirmation that I wasn't ruining it. (usual care 9 )

Others gave examples about how the content of therapy and targets were regulated by their therapist.

When she come she used to give me target. Like for instance she would say I'm giving you this target, do this, 
do this, do this and when I come I will re-examine you how much you have achieved. How much you have come far. (sic) (usual care 10)

Despite the importance of following advice by some therapists, there was also acknowledgement in some accounts about how usual care participants' had ended up doing in things in their own way, using therapists ideas but constructing a way forward independently.

I don't think they're happy really. But at the end of the day they don't ask me to stop doing it. The only thing they did say is when you stand up don't pull. Don't use it as a leverage. Just use it as a guide. And I do. Well we'll see how it goes. (usual care 13)

\section{What happens in the future?}

Uncertainty about future progress was prevalent across all transcripts but was construed in different ways. While some participants expressed concerns about a lack of direction there was recognition by all that therapy would stop at some point. Our topic guide specifically explored how participants felt about managing when therapy stopped as questioned by 'how confident do you feel to continue under your own steam?' Many responses depicted apprehension but there was self-confidence expressed only by participants in the integrated selfmanagement sites.

The only thing I don't know about now is what the future holds for me. Because I know they [therapists] cannot go on like this forever but... (usual care 20).

As I was saying to [Physiotherapist] the other day, when are you people off? And she said when you feel like you don't need us anymore. (integrated self-management 4)

There was also a range of responses to the question 'if you had a problem after discharge what would you do?'

No, I'd have a go myself. Why should I contact them? They've got a lot of people to work with. I wouldn't want to be a bother? (integrated self-management 5)

I would see it (finishing therapy) as a challenge you see. And if I feel something is a challenge, I will try to see if I can overcome that problem (integrated selfmanagement 4)

I just want some confirmation that I am doing the right thing. A back up, yeh that's the only thing (usual care 2)

Overall, this subtheme suggests varying levels of concern about the future and what happens after discharge from rehabilitation, but the uncertainty was not seen as negative by all participants. Each of the subthemes above illustrate a variety of ways in which control and responsibility for forward progress have been perceived by participants. Differences in accounts were revealed when exploring issues such as "who controls the content of therapy' and 'confidence to manage after discharge'. The next theme relates to how knowledge and resources (from therapist and stroke participants) were used to varying degrees within rehabilitation.

\section{Theme 2-knowledge and resources}

This theme depicts varying ways in which participants described how their past skills were given priority and meaning, including the ways in which they had been supported to contribute their own solutions and create expertise together with their therapist. The theme is comprised of subthemes; 'utilising past skills' which was depicted only within accounts from integrated selfmanagement interviews and 'building knowledge together' and the 'benefit of a close relationship' which was generated from all interviews.

\section{Utilising past skills}

The transference of past skills and experiences towards managing health is often advocated in approaches to self-management support and some participants described how their therapist had encouraged them to apply their past skills to advance their progress.

One participant talked about the realisation of when his previous skills could align with the skills he needed to cope post stroke.

I suddenly sat down and thought, "well which project what am I going to do today?' And I planned it day by day and I thought, 'hang on this is like being back at work' and everything started clicking. (integrated selfmanagement 1)

Experimentation and adaptation was visible alongside reports of encouragement given by therapists to try things out. There was a sense held by some participants of having a go and not feeling worried if it failed.

I mean you have to adapt things. All of a sudden I found myself adapting in ways of putting on my socks or. Well I did building maintenance before so I would adapt things. So thinking, 'ok instead of doing it like this I have to do it like this.' (integrated self-management 2)

I found a way of doing it, it may not always be the 'correct way', but as long as it was a safe way then they were with it, absolutely. (integrated self-management-14)

\section{Building knowledge together}

Participants talked about how they gained a gradual familiarity and knowledge about how to cope with different challenges which arose from a subtle process of sharing ideas and having their contribution confirmed.

They did ask [for my ideas]. Yes. they did ask me. And I said I was alright because he said come out of the kitchen and then you can hold onto the side if you can. And then I said to him 'I do something a bit different' and he watched and he said 'you do it your way. You 
seem to be doing it better your way'. (integrated selfmanagement 16)

The relationship was supportive and enabling in some instances and participants felt valued and were encouraged to ask for advice.

She says to me how do you feel about this? Not 'do this'. You see quite a clear distinction between them isn't it? She's not saying you do this. She's saying how do you feel about doing this? (integrated self-management 4)

There was a distinct emphasis given by some participants that the therapist was there for them, as an aid to their needs gradually helping them to build expertise.

It was good to know that I was able to be in control. And she just gave me the assurance that this was right for the stroke rather than right for me, which was important. (integrated self-management 7)

\section{Benefit of a close relationship}

Participants across all interviews focused on the importance of the (often close) relationship and the guidance and support they had received at key times.

You need someone, you've never met this person before, they're coming into your home or whatever and you are seeing them when you are feeling like crap, you don't want to do anything, you look like shit so you kind of just want somebody you can talk to. (usual care 5)

All participants described their relationship with therapists to be supportive and open, and gave examples of how their efforts were generally encouraged. Some participants appreciated how therapists validated their own ideas and ways to do things. 'Because sometimes when they come if I'm tired, maybe I did domestic, or hoovering and I thought I don't want to do it this way and that I feel more confident in doing it a different way- they would say, oh that's ok do it in a way that you feel confident in doing'. (integrated self-management 17)

In addition, the expertise and importance of following the advice of therapists was acknowledged by many participants.

They were very experienced you could see that so it wasn't one way...I mean if I didn't have them I don't know how I would have coped. Whether you would have tried to do it yourself and just made it worse. (usual care 21)

Overall, the knowledge and resources highlighted by participants often related to the skills and support provided by therapists. But participants from the integrated self-management group also highlighted their own resources which had emerged and been encouraged by therapists. The time limited nature of rehabilitation seemed to be prominent in most participants' minds but the impact of stopping and managing without therapy was perceived in different ways. Positively framed responses about the prospect of 'continuing under your own steam' came almost entirely from the integrated self-management group.

\section{DISCUSSION}

This paper reports on findings from qualitative interviews which aimed to explore (1) whether the experiences and accounts from stroke survivors receiving rehabilitation in usual care and integrated selfmanagement groups reflected the differences in the therapy they had received and (2) the extent to which their experiences aligned with some or any of the key principles of the self-management approach used.

All participants interviewed revealed a shared appreciation of knowledge and support of therapists but some subtle differences emerged between sites. A greater extent participants in the integrated self-management group shared different perceptions about responsibility, control and how their own previous experiences and ideas were used. Our logic model assumed that integrated self-management group participants having received rehabilitation with a focus on self-efficacy and self-management might depict their rehabilitation differently to those receiving other forms of community stroke rehabilitation (usual care). Overall, this was found to be the case with some variations. A central finding was that all participants recalled how rehabilitation plans were introduced, success was measured and feedback given. But accounts depicted a variance between participants regarding who had structured and planned their rehabilitation, with greater flexibility about content and involvement in plans perceived by integrated selfmanagement site participants. Moreover, participants from integrated self-management sites provided demonstrable evidence that their experiences aligned with some of the principles of the SMP integrated selfmanagement, such as self-discovery and problem-solving (box 1).

While research consistently supports the need for more stroke rehabilitation, it remains a time limited resource. $^{2728}$ Our research suggests that not all participants felt a sense of despair when therapy finished as previously noted by different authors ${ }^{29}$ and the reasons for this require further exploration. This seems to suggest that providing a shared platform for collaboration from the start of therapy could make the transition to discharge less of a problem, as described in results one integrated self-management participant recalled saying to the therapists 'when are you lot off then?', he was keen to be discharged and get on with his life, ultimately a successful outcome for him and the rehabilitation team.

A common aspect of many self-management programmes is the process of individuals building knowledge together with a healthcare professional. This is a component of what is termed 'shared decision-making' 
a critical facet of patient-centred practice. ${ }^{30}$ In the context of stroke rehabilitation this would require professionals to relinquish the lead in planning and structuring rehabilitation and take a more flexible approach. There are several barriers to this as noted by different authors, not least that this approach is considered more time-consuming and professionals raise concerns that patients might not have the skills to lead and make decisions about their rehabilitation. ${ }^{7-9}$ Therapists using an integrated self-management approach in this study were trained to focus specifically on providing opportunities for devising goals together and encouraging individuals to come up with their own ideas, reflecting and problem-solving about what had or had not worked and why. Therapists were encouraged to find ways to incorporate mastery experiences in every therapy session, a key source of self-efficacy. ${ }^{22}$ Attributing success to individual efforts and reducing the focus on expertise of professionals can strengthen individual self-efficacy and has been associated with improved outcomes post stroke. ${ }^{31-}$ ${ }^{33}$ We found that participants receiving rehabilitation with an emphasis on mastery experiences and joint problem-solving tended to acknowledge and reflect on their own expertise to a greater degree. As such, therapy expertise was seen as an important source of information and knowledge but not the only source of ideas. This suggests that stroke survivors can appreciate receiving therapy which facilitates opportunities to use their past skills, create plans together with therapists and try out ideas. Equally they value the support and knowledge held and given by therapists.

This study also enabled an opportunity to explore the meaning of self-management with participants without using the term directly, which was a useful strategy to gain an understanding of how they described rehabilitation and responsibility for rehabilitation. An approach which comprises a continuum of self-management support by therapists could be preferable given that stroke survivors have faced a sudden and often extreme life event and may require greater direction and support in the acute stages. Despite acknowledging the importance of self-efficacy as an underpinning construct, we would concur with other researchers that selfmanagement support which promotes collective responsibility rather than an exclusive focus on individual responsibility is preferable. ${ }^{34}$ Self-management continues to be defined in much of UK health policy with a focus on personal agency and behaviour change which we believe could be counterproductive and alienating, if a person is perceived by professionals as unwilling to engage or not motivated to self-manage. ${ }^{34}{ }^{35}$ Stroke survivors experience a complex range of issues including emotional and cognitive difficulties and could find the term self-management disaffecting. As others have commented, there will be individuals who do not or cannot self-manage and a 'one-size approach' may compound the sense of being left to get on with things after rehabilitation stops. ${ }^{25}$ This requires sensitivity and skills on the part of a therapist to recognise and tailor selfmanagement using methods which are inclusive to more individuals. Equally, therapists were taught in this study to recognise that a reluctance to engage and reliance on professional expertise may not reflect a lack of desire for stroke survivors to be involved. Moreover, perceived passivity on the part of stroke survivors may reflect the result of minimal opportunities to build confidence and individual psychosocial characteristics which could influence learning and engagement.

We believe that findings from this study could inform the incremental development of an integrated stroke self-management programme. We were keen to explore the mechanisms relating to the intended outcome of the integrated self-management through the accounts of participants from usual care and integrated selfmanagement sites and explore whether there were any potential unintended consequences. Overall, we found very little reference to the idea of 'self-management' but we deliberately set out to explore the practices which could be construed as self-management rather than the term per se. Participants from the integrated selfmanagement groups did not perceive a self-management approach to be burdensome, but these findings have to be taken in the context of a feasibility trial of a complex integrated self-management intervention, and therapy received in the usual care sites would also have many complexities and interacting components. We found that none of the study participants felt they were burdened by rehabilitation and the demands of therapy and all acknowledged the close and supportive relationship they had with their therapist and the ideas they had gained.

\section{STRENGTHS AND LIMITATIONS}

We fully acknowledge that interviews were carried out at one time point, the views and experiences of participants could change and the findings provide no indication of any long-term impact. We consecutively recruited stroke survivors for interviews from a larger sample already screened and recruited for a feasibility trial. We acknowledge that as with many other stroke studies participants with aphasia were under-represented. Future studies could use different methods of evaluation which do not rely so heavily on spoken language such as visual representation of experiences, for example, Talking Mats. ${ }^{36}$ We are also aware of the potential for bias in the assumptions made by those involved in the data collection and analysis. While attempts were made to maintain reflexivity and rigour during the research, prior understanding of self-management and rehabilitation held by the researchers carrying out the interviews may have shaped the process to some degree. We included a number of steps to mitigate for potential sources of bias. One strategy was to share our preliminary findings with an independent stakeholder group of stroke survivors involved in developing the interview topic guide. This 
provided us with a clear format for the interviews without making specific reference to the integrated selfmanagement intervention until the very end of the interview when we asked about use of the stroke workbook. We also shared initial transcripts with other researchers from a non-clinical background to screen for consistency in questions and contribute their ideas to preliminary analysis.

Primarily, we set out to explore experiences of participants across all sites and the meaning given to rehabilitation. But we also wanted to learn the extent to which experiences, strategies and activities depicted in their accounts aligned with self-management. We felt that the study would be strengthened by acknowledging how rehabilitation was constructed differently in some areas by making reference to whether the data contributing to subthemes were from integrated self-management or usual care sites. We are aware of the obvious limitations in carrying out qualitative research in which the interviewers know whether participants have received the integrated self-management that is being studied. While the focus on feasibility and early exploration of mechanisms of implementation could justify our approach, in hindsight we feel that findings would have been strengthened by using researchers who were blinded to the allocation of participants and we would recommend this in a larger definitive trial. Equally, we recognise that many other contextual factors such as service and team configuration may have influenced participants' experience of rehabilitation and these factors are important to capture through different methods such as non-participant observation in any future trials. The impact of the integrated self-management intervention based on these findings cannot be inferred and we recognise that interview data are only one aspect of understanding fidelity of an intervention and the underlying mechanisms of action.

\section{CONCLUSION}

The complexity of stroke has driven the need for exploring and testing different ways of offering selfmanagement support, and integrating interventions into existing rehabilitation could offer a way forward. Our findings partly reflect our theoretical assumptions and possible mechanisms of implementation, that therapy focused on supporting self-efficacy and self-management could be reflected in accounts of stroke survivors. But further questions about how an integrated selfmanagement intervention is enacted by rehabilitation teams and the impact on key outcomes relating to confidence, mood and functional capability is required to gain a full understanding of the intervention theory. Nonetheless, we believe that the findings from this process evaluation can inform future design and testing of integrated self-management programmes and contribute to an understanding of methods to capture how self-management programmes are perceived by stroke survivors at different stages of their rehabilitation.

It is imperative to develop self-management approaches which are tailored and sensitive to the complexity of stroke while not being perceived as a burden by either stroke survivors or professionals. Nevertheless, an integrated self-management approach such as Bridges SMP requires professionals to be critically aware of the language and strategies used in rehabilitation sessions and how they can potentially foster and enable confidence to self-manage. Studies testing integrated approaches should incorporate multiple methods to explore and evaluate fidelity and impact including how the intervention is perceived and understood by stroke survivors. These findings taken together with our previous research justifies the value of further evaluating the impact and effectiveness of Bridges stroke SMP in a larger sample to extend our understanding of the fidelity of the approach, contextual factors that could impact on implementation, and possible mechanisms of impact.

\section{Author affiliations}

${ }^{1}$ Faculty of Health, Social Care and Education, Kingston University and St George's, University of London, London, UK

${ }^{2}$ National Institute for Health Research (NIHR), Collaboration for Leadership in Applied Health Research and Care South London, London, UK

${ }^{3}$ Division of Health \& Social Care Research, Faculty of Life Sciences \&

Medicine, King's College London, London, UK

${ }^{4}$ National Institute for Health Research (NIHR), Biomedical Research Centre at Guy's and St Thomas' NHS Foundation Trust and King's College London,

London, UK

${ }^{5}$ Department of Psychology, Royal Holloway University of London, London, UK

${ }^{6}$ School of Science and Health, University of Western Sydney, Australia

Acknowledgements We acknowledge the input from our advisory groups: support from the Collaboration for Leadership in Applied Health Research and Care (CLAHRC) South London and Dr Stefan Tino Kulnik for his help with later drafts of this paper. We also acknowledge the contribution of Siân Jones and Carmen Lech with preliminary coding of data.

Contributors All authors designed the study. FJ, CMc, AR and ML performed data analysis. FJ, CMc and AR wrote initial draft and all authors commented and contributed to the manuscript.

Funding This report presents independent research funded by the National Institute for Health Research (Research for Patient Benefit Programme; grant number: PB-PG-0610-22276).

Disclaimer The views expressed in the publication are those of the authors and not necessarily those of the NHS, the National Institute for Health Research or the Department of Health.

Competing interests $\mathrm{FJ}$ is the founder and director of the social enterprise Bridges self-management.

Ethics approval The London Surrey Borders National Research Ethics Committee gave ethical approval for this study (11/LO/1450) with local research and development approval granted from each of the cluster sites.

Provenance and peer review Not commissioned; externally peer reviewed.

Data sharing statement Patient level data (qualitative semistructured interviews) are available from the corresponding author. Informed consent for data sharing was not obtained, but presented data are anonymised and risk of identification is very low.

Open Access This is an Open Access article distributed in accordance with the Creative Commons Attribution Non Commercial (CC BY-NC 4.0) license, which permits others to distribute, remix, adapt, build upon this work non- 
commercially, and license their derivative works on different terms, provided the original work is properly cited and the use is non-commercial. See: http:// creativecommons.org/licenses/by-nc/4.0/

\section{REFERENCES}

1. Corbin JM, Strauss AL. Unending work and care: managing chronic illness at home. Jossey-Bass Publishers, 1988.

2. Parke HL, Epiphaniou E, Pearce G, et al. Self-management support interventions for stroke survivors: a systematic meta-review. PLoS ONE 2015;10:e0131448.

3. Boden-Albala B, Litwak E, Elkind MSV, et al. Social isolation and outcomes post stroke. Neurology 2005;64:1888-92.

4. Salter K, Hellings $\mathrm{C}$, Foley N, et al. The experience of living with stroke: a qualitative meta-synthesis. J Rehabil Med 2008;40:595-602.

5. Ayerbe L, Ayis S, Rudd AG, et al. Natural history, predictors, and associations of depression 5 years after stroke. The South London Stroke Register. Stroke 2011;42:1907-11.

6. Norrving B, Kissela $B$. The global burden of stroke and need for a continuum of care. Neurology 2013;80:S5-12.

7. Levack WM, Dean SG, Siegert RJ, et al. Navigating patient-centered goal setting in inpatient stroke rehabilitation: how clinicians control the process to meet perceived professional responsibilities. Patient Educ Couns 2011;85:206-13.

8. Norris M, Kilbride C. From dictatorship to a reluctant democracy: stroke therapists talking about self-management. Disabil Rehabil 2014;36:32-8.

9. Mudge S, Kayes N, McPherson K. Who is in control? Clinicians view on their role in self-management approaches: a qualitative metasynthesis. BMJ Open 2015;5:e007413.

10. Rosewilliam S, Roskell C, Pandyan A. A systematic review and synthesis of the quantitative and qualitative evidence behind patient-centred goal setting in stroke rehabilitation. Clin Rehabil 2011;25:501-14.

11. de Silva D. Helping people help themselves: a review of the evidence considering whether it is worthwhile to support self-management. London: The Health Foundation, 2011. http://www.health.org.uk/ media_manager/public/75/publications_pdfs/Helping\%20people\% 20help\%20themselves.pdf (accessed 15 Sep 2015).

12. Lennon S, McKenna S, Jones F. Self-management programmes for people post stroke: a systematic review. Clin Rehabil 2013;27:867-78.

13. Coulter A, Ellis J. Effectiveness of strategies for informing, educating and involving patients. The BMJ 2007;335:24-7.

14. Fryer CE, Luker JA, McDonnell MN, et al. Self management programmes for quality of life in people with stroke. Cochrane Database Syst Rev 2016;(8):CD010442.

15. Cadilhac D, Kilkenny MF, Srikanth V, et al. Do cognitive, language, or physical impairments affect participation in a trial of self-management programs for stroke? IntJ Stroke 2016;11:77-84.

16. State of the Nation Stroke statistics. https://www.stroke.org.uk/sites/ default/files/stroke_statistics_2015.pdf (accessed 28 Aug 2016).

17. Pederson PM, Jørgensen $\mathrm{HS}$, Nakayama $\mathrm{H}$, et al. Aphasia in acute stroke: incidence, determinants, and recovery. Ann Neurol 1995;38:659-66.
18. An Implementation Guide and Toolkit for Making Every Contact Count: Using every opportunity to achieve health and wellbeing. https://www.england.nhs.uk/wp-content/uploads/2014/06/ mecc-guid-booklet.pdf (accessed 28 Aug 2016).

19. Jones F, Brimicombe L. Every interaction counts: the 'Bridges' approach to stroke self-management. Int $J$ Ther Rehabil 2014;21:158-9.

20. McKenna S, Jones F, Glenfield P, et al. Bridges self-management programme for people with stroke in the community: a feasibility randomised controlled trial. Int J Stroke 2015;10:697-704.

21. Jones F, Gage H, Drummond A, et al. Feasibility study of an integrated stroke self-management programme: a cluster-randomised controlled trial. BMJ Open 2015;5:e008900.

22. Bandura A. The nature and structure of self-efficacy. In: Bandura A, ed. Self-efficacy: the exercise of control. New York, NY: WH Freeman and Company, 1997:37-78.

23. Craig P, Dieppe P, Macintyre S, et al. Medical Research Council Guidance. Developing and evaluating complex s: the new Medical Research Council guidance. BMJ 2008;337:a1655.

24. Moore G, Audrey S, Barker M, et al. Process evaluation of complex interventions: Medical Research Council guidance. London: MRC Population Health Science Research Network, 2014. http://www. populationhealthsciences.org/ MRC-PHSRN-Process-evaluation-guidance-final-2-.pdf (accessed 28 Aug 2016).

25. Satink T, Cup EH, de Swart BJ, et al. How is self-management perceived by community living people after stroke. Disab Rehabil 2015;37:223-30.

26. Braun V, Clark V. Using thematic analysis in psychology. Qual Res Psychol 2006;3:77-101.

27. Stroke Association: Struggling to Recover, Life After Stroke Campaign Briefing. https://www.stroke.org.uk/sites/default/files/ struggling_to_recover_report_lowres.pdf (accessed 28 Aug 2016).

28. Langhorne P, Bernhardt J, Kwakkel G. Stroke rehabilitation. Lancet 2011;377:1693-702.

29. Wiles R, Ashburn A, Payne S, et al. Discharge from physiotherapy following stroke: the management of disappointment. Soc Sci Med 2004;59:1263-73.

30. Légaré $P$, Thompson-Luduc $P$. Twelve myths about shared decision making. PatEduc Couns 2014;96:281-6.

31. Jones F, Riazi A, Norris M. Self-management after stroke: time for some more questions? Disabil Rehabil 2013;35:257-64.

32. Korpershoek C, van der Bijl J, Hafsteinsdottir TB. Self efficacy and its influence on recovery of patients with stroke: a systematic review. $J$ Adv Nur 2011;67:1876-94.

33. Demain S, Burridge J, Ellis-hill C, et al. Assistive technologies after stroke: self-management or fending for yourself? A focus group study. BMC Health Serv Res 2013;13:334

34. Boger E, Ellis J, Latter S, et al. Self-management and self-management support outcomes: a systematic review and mixed research synthesis of stakeholder views. PLOS ONE 2015;10: e0130990.

35. Vassilev I, Rogers A, Kennedy A, et al. The influence of social networks on self-management support: a metasynthesis. BMC Public Health 2014;17:719.

36. Murphy J. Enabling people with aphasia to discuss quality of life. $\mathrm{Br} J$ Ther Rehabil 2000;7:454-8. 